\title{
Nosema spp. infections cause no energetic stress in tolerant honeybees
}

\author{
Christoph Kurze $^{1 *}$, Christopher Mayack ${ }^{1}$, Frank Hirche ${ }^{2}$, Gabriele I. Stangl ${ }^{2}$, \\ Yves Le Conte $^{3}$, Per Kryger ${ }^{4}$, Robin F. A. Moritz ${ }^{1,5,6}$ \\ ${ }^{1}$ Institute for Biology, Martin-Luther-Universität Halle-Wittenberg, 06099 Halle (Saale), Germany \\ ${ }^{2}$ Institute of Agricultural and Nutritional Sciences, Martin-Luther-Universität Halle-Wittenberg, 06120 Halle \\ (Saale), Germany \\ ${ }^{3}$ INRA, UR 406 Abeilles et Environnement, 84914 Avignon, Cedex 9, France \\ ${ }^{4}$ Department of Agroecology Flakkebjerg, Aarhus University, 4200 Slagelse, Denmark \\ ${ }^{5}$ German Institute for Integrative Biodiversity Research (iDiv), 04103 Leipzig, Germany \\ ${ }^{6}$ Department of Zoology and Entomology, \\ *Correspondence to: * Christoph Kurze, christoph.kurze@ zoologie.uni-halle.de
}

\begin{abstract}
Host-pathogen coevolution leads to reciprocal adaptations, allowing pathogens to increase host exploitation or hosts to minimise costs of infection. As pathogen resistance is often associated with considerable costs, tolerance may be an evolutionary alternative. Here, we examined the effect of two closely related and highly host dependent intracellular gut pathogens, Nosema apis and Nosema ceranae, on the energetic state in Nosema tolerant and sensitive honeybees facing the infection. We quantified the three major haemolymph carbohydrates fructose, glucose, and trehalose using high-performance liquid chromatography (HPLC) as a measure for host energetic state. Trehalose levels in the haemolymph were negatively associated with $N$. apis infection intensity and with $N$. ceranae infection regardless of the infection intensity in sensitive honeybees. Nevertheless, there was no such association in Nosema spp. infected tolerant honeybees. These findings suggest that energy availability in tolerant honeybees was not compromised by the infection. This result obtained at the individual level may also have implications at the colony level where workers in spite of a Nosema infection can still perform as well as healthy bees, maintaining colony efficiency and productivity.
\end{abstract}

\section{Keywords}

Host-parasite interaction Immune response Energetic stress Adaptation Fitness cost

\section{Introduction}

An infection or disease can lead to a severe reduction of host fitness by reducing both host survival and reproductive success (Hurd, 2001). These fitness costs to the host are not only imposed by pathogens directly but may also be a result of immune function itself (Lochmiller $\&$ Deerenberg, 2000; Schmid-Hempel, 2005). The host has to reallocate its resource availability investing more into immune responses which typically leads to life-history tradeoffs (Sheldon \& Verhulst, 1996). For example, an immune challenge may result in decreased survival (Moret \& Schmid-Hempel, 2000), self-harm (Sadd \& Siva-Jothy, 2006), or altered development and fecundity (Roth \& Kurtz, 2008). Because immunocompetence is a costly process (Schmid-Hempel, 2005), natural selection would not necessarily favour fully resistant 
host phenotypes, but rather tolerant hosts that can sustain parasitic infection, keeping infection intensity at bay while sustaining a high level of fitness (Råberg et al., 2007; Sorci, 2013). Thus, a well-adapted host might be able to either allocate resources more efficiently or has acquired a mechanism to compensate for any resource losses due to parasitic infection. In social insect societies such as honeybees, sterile workers contribute to the fitness of the colony without receiving direct fitness benefits (Hamilton, 1964; Moritz \& Southwick, 1992). Hence, it is the fraction of infected workers in a colony which is a critical parameter in determining tolerance to an infectious disease rather than the infection level of an individual worker (Straub et al., 2015).

In antagonistic coevolution, pathogens will be selected to minimise the cost of immune clearance and counter-adapt by either escaping or at least reducing the effectiveness of the host's immune system (Schmid-Hempel, 2008). Escaping the host's immune response might be particularly important for highly specialised parasites such as Microsporidia. Because this group of intracellular fungi lacks mitochondria, they are entirely dependent on the host's energy metabolism to ensure reproduction and survival (Keeling, 2009). Microsporidia have evolved elaborate infection mechanisms in a wide range of animal hosts, which includes humans and in economically and ecologically important animals such as honeybees, silk worms, and salmon (Keeling, 2009). In particular the emerging gut pathogen Nosema ceranae has gained tremendous attention in recent years and is considered to be an important pathogen in honeybees worldwide (Higes et al., 2013). Similar to its native closely related sister species Nosema apis, spores of $N$. ceranae are horizontally transmitted by ingestion and germinate in the host's ventricular (midgut), where they penetrate and enter epithelial cells via their polar tubes. Nosema then exploits the host cell's metabolism for its own reproduction (Fries, 2010; Gisder et al., 2011).

Although $N$. ceranae infection does not appear to alter the navigation and orientation of the honeybees while foraging, increased foraging duration and decreased flight frequencies have been reported in infected honeybees (Alaux et al., 2014; Dussaubat et al., 2013; Naug, 2014; Wolf et al., 2014). This decline in foraging efficiency is likely to be a result of imposed nutritional and energetic stress from infection (Aliferis et al., 2012; Martín-Hernández et al., 2011; Mayack \& Naug, 2009; Mayack \& Naug, 2010; Moffett \& Lawson, 1975). Infected honeybee workers also appear to gain energy less efficiently, which may negatively influence their lifespan and the colony performance on a long-term basis (Naug, 2014). Moreover, $N$. ceranae infection causes immunosuppression in honeybees (Antúnez et al., 2009; Chaimanee et al., 2012; Holt et al., 2013; Huang et al., 2012), which could be the consequence of energetic stress due to costly maintenance of an immune response (Schmid-Hempel, 2005).

Because Nosema infections are negatively associated with honeybee health, Danish honeybee queen breeders selected against Nosema spp. by excluding infested queens from further breeding for two decades (Hatjina et al., 2014). Not only did the Nosema spp. prevalence decline from 60-80\% to approximately $10 \%$ in colonies of this tolerant strain (based on 60 workers/colony) as a result of this artificial selection (Hatjina et al., 2014), but moreover these tolerant honeybees appeared to survive $N$. ceranae infection better than Nosema sensitive honeybees, which could be explained by up-regulated expression levels of key immune genes (Huang et al., 2012). However, it is not known whether these selected tolerant honeybees suffer trade-offs due to resource allocation shifts. Although an increase immune response may cause additional nutritional or energetic costs to the host (Schmid-Hempel, 2005), tolerance is a mechanism allowing the host to sustain the infection and reduce the 
damage induced by pathogens or overreacting immune response while paying little or even no fitness costs (Råberg et al., 2007; Sorci, 2013).

Therefore, we use haemolymph carbohydrates as an absolute measure of energy allocation and availability during the infection in this study. We compared the energetic cost associated with a Nosema infection between a Nosema tolerant and sensitive honeybee lineage. In honeybees, the disaccharide trehalose circulating in the haemolymph acts as a major energy storage source to fuel activities such as flight (Blatt \& Roces, 2001; Suarez et al., 1996). In addition, the carbohydrate composition in the haemolymph reflects nutrient intake and can be used as an indicator of nutritional status in insects (Thompson, 2003). We therefore decided to measure the concentration of trehalose, glucose and fructose in the haemolymph to estimate the energetic costs incurred from a Nosema infection. We hypothesise that energy availability in the haemolymph is negatively associated with Nosema infection load in Nosema sensitive honeybees. In addition, we hypothesise that Nosema tolerant honeybees in contrast to sensitive honeybees compensate any energy depletion via to an adaptation of their energy metabolism to Nosema spp. infection.

\section{Materials and methods}

\section{Honeybee hosts}

Worker honeybees (Apis mellifera) were first reared from the selected Nosema tolerant (T) lineage, provided by the Department of Integrated Pest Management Research Centre Flakkebjerg, Denmark, and from a Nosema sensitive (S) lineage, provided by the Laboratoire des Abeilles et Environnement de l'INRA Avignon, France. Frames of capped worker brood were obtained from each lineage and kept in an incubator at $34 \pm 1{ }^{\circ} \mathrm{C}, 60 \%$ relative humidity and $24 \mathrm{~h}$ darkness. Newly emerged Nosema free worker honeybees ( $<12 \mathrm{~h}$ old) were collected from brood frames. They were housed in groups of 30 individuals each in autoclaved stainless steel cages $(10 \times 10 \times 5.5 \mathrm{~cm})$ and placed back into the incubator. Throughout the entire duration of the trials, honeybees were fed $50 \%(w / v)$ sucrose solution ad libitum.

\section{Nosema spp. isolates}

For inoculations, we used $N$. ceranae spores originating from Germany and $N$. apis spores originating from Sweden. N. ceranae spores were freshly extracted and N. apis spores were obtained from frozen stock (Fries, 2010). Inocula were then purified using the triangulation centrifugation method (Fries et al., 2013). To obtain the required Nosema concentration, we counted Nosema spores using a Fuchs-Rosenthal haemocytometer placed under a phasecontrast microscope (Olympus CX41, Olympus, Hamburg, Germany) and then diluted spores to a concentration of approximately 50,000 spores $\mu^{-1}$ in $50 \%(w / v)$ sucrose solution before inoculation. Nosema species identification was confirmed by PCR as described previously (Gisder \& Genersch, 2013).

\section{Experimental inoculation}

Inoculation experiments were conducted for each honeybee lineage $(\mathrm{S}=$ sensitive and $\mathrm{T}=$ tolerant) at the Martin-Luther-Universität Halle-Wittenberg in Germany from May to July 2014, following standard methods for Nosema research in honeybees (Fries et al., 2013). Three day old bees were either individually fed with approximately $10^{5} \mathrm{~N}$. ceranae (SNc and 
$\mathrm{TNc}$ ) or $N$. apis (SNa and TNa) spores in $2 \mu \mathrm{l}$ of $50 \%(w / v)$ sucrose solution to induce successful infections $\left(\right.$ ID $_{100}$ ) (Forsgren \& Fries, 2010; Fries, 2010). Negative controls (SC and TC) were individually fed with $2 \mu$ of pure sucrose solution. Only bees consuming the entire inoculum were included in the experiments. We chose to terminate the experiment at a relatively early stage of the infection on 6 days post infection (p.i.), when infection had been established and the first generation of spores had been produced (Fries et al., 1992; Gisder et al., 2011), to obtain individuals facing infections ranging from low to high intensities and to make our results comparable with previous experiments (Huang et al., 2012; Kurze et al., 2015). Haemolymph was obtained from approximately five randomly chosen individuals per treatment group per replicate, and the digestive tract was immediately dissected out of the bee. Four replicates were conducted per treatment group, except for $N$. apis infected tolerant honeybees with only three replicates.

\section{Haemolymph extraction}

Haemolymph collection was performed according to standard methods (Hartfelder et al., 2013). Briefly, bees were immobilised by crossing two pins over the waist of the bee mounted on a wax-filled Petri dish. Then, $2 \mu$ of haemolymph was collected from a small incision on the side of the bee's abdomen using a glass microcapillary (Brand, Wertheim, Germany). Obtained haemolymph was subsequently snap frozen in liquid nitrogen and then stored at $-80^{\circ} \mathrm{C}$ until HPLC analysis.

\section{Nosema spore count}

Both $N$. ceranae and N. apis are known to only infect the ventriculus (i.e. midgut without rectum) tissue of the honeybee (Huang \& Solter, 2013). Thus, midguts of at least five randomly chosen bees per treatment group and replicate were individually homogenised in $1 \mathrm{ml}$ distilled water and the numbers of mature spores were counted with a Fuchs-Rosenthal haemocytometer placed under a phase-contrast microscope $(\times 400)$.

\section{Determination of sugar levels}

For the identification and quantification of the three sugars found in honeybee haemolymph (glucose, fructose, and trehalose), we used high-performance liquid chromatography (HPLC). In addition, sucrose was measured to serve as an indicator of crop contamination which might have occurred during the haemolymph extraction. Prior to HPLC analysis, $2 \mu \mathrm{l}$ of each haemolymph sample were mixed with $22 \mu$ acetonitrile $\left(\mathrm{CH}_{3} \mathrm{CN}\right)+$ distilled water $(8+2 v / v)$, kept on ice and centrifuged at 10,000 g for 6 min maintained at $4{ }^{\circ} \mathrm{C}$ (Eppendorf centrifuge 5804 R, Eppendorf, Germany) (Bozic \& Woodring, 1997).

Then $20 \mu \mathrm{l}$ of the final supernatant per sample was transferred to microvials with glass inserts and subsequently $10 \mu 1$ was separated at $30^{\circ} \mathrm{C}$ constant temperature using a 5 - $\mu \mathrm{m}$ Nucleosil$100 \mathrm{NH}_{2}$ column $\left(250 \times 4 \mathrm{~mm}^{2}\right.$, Knauer, Berlin, Germany $)$ in the HPLC Agilent 1100 series system with autosampler (Agilent Techologies, Waldbronn, Germany). The flow rate was set

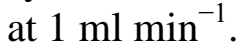

For separation of carbohydrates, the following elution programme based on (A) acetonitrile + water $(87+13 \mathrm{v} / \mathrm{v})$ and $(\mathrm{B})$ acetonitrile + water $(50+50 \mathrm{v} / \mathrm{v})$ was used: $0 \mathrm{~min}$ $100 \%$ (A), $10 \min 87 \%$ (A), 18 min $80 \%$ (A), 19 min $50 \%$ (A), 23 min $50 \%$ (A), 24 min $100 \%$ (A), and 30 min $100 \%$ (A). The system was calibrated after every 20 samples using at 
least five levels of standard solutions containing a mixture of trehalose, glucose, fructose, and sucrose at varying concentrations to cover the entire biological realistic range of possible sample concentrations.

Detection of carbohydrates was accomplished with a SEDEX 55 evaporative light-scattering detector (S.E.D.E.R.E., Altertville Cedex, France). The detector settings were kept constant throughout the HPLC analyses, the evaporation temperature was set at $75^{\circ} \mathrm{C}$ and the nitrogen pressure at 1.9 bar. Data were acquired and analysed with the ChemStation A08.03 software (Agilent Technologies). All results were converted into $\mathrm{mg} \mathrm{ml}^{-1}$. If a sample contained more than $0.5 \mathrm{mg} \mathrm{ml}^{-1}$ sucrose, this was considered as a sign of contamination and these samples were not included in the analysis (Woodring et al., 1993).

\section{Statistical analysis}

Statistical analyses and data plotting were performed in $\mathrm{R}$ version 3.2.1 (R Development Core Team, 2015). The numbers of $N$. apis and $N$. ceranae spores were $\log _{10}$ transformed for all statistical tests. Differences in spore loads between treatment groups were tested using a Kruskal-Wallis one-way ANOVA, followed by pairwise comparisons using the Wilcoxon rank sum test. Multiple testing was accounting with Bonferroni adjustments. Depending on the data distribution, we tested the effect of treatment and honeybee lineages and their interactions on haemolymph sugar level, using a Kruskal-Wallis one-way ANOVA for glucose and fructose and a two-way ANOVA for trehalose levels. Post hoc analyses between treatment groups were performed using the glht function with the Bonferroni adjustment (multcomp package, v.1.4-1). Linear regression analyses were computed between the $\log _{10}$ number of Nosema spp. spores and the haemolymph trehalose concentrations for Nosema sensitive and tolerant honeybees. Differences between slopes were calculated by using MANCOVA. To avoid any statistical bias, we inspected residuals, leverage and Cook's D and tested for potential outliers with Bonferroni correction.

\section{Results}

\section{Infection load}

The mean $\log _{10}$ number of spores \pm standard deviation (s.d.) in sensitive and tolerant honeybees were $5.46 \pm 0.82(\mathrm{SNa}, n=20)$ and $4.73 \pm 0.76(\mathrm{TNa}, n=15)$, respectively, for $N$. apis infections and $5.61 \pm 0.42(\mathrm{SNc}, n=22)$ and $5.23 \pm 0.83(\mathrm{TNc}, n=22)$, respectively, for $N$. ceranae infections. All screened controls $(\mathrm{SC}, n=20 ; \mathrm{TC}, n=23)$ were free of Nosema spp. infection. Although there was a significant effect between treatment groups (KruskalWallis: $\chi^{2}=11.675, \mathrm{df}=3, p=0.01$ ), where the $\log _{10}$ spore load in SNc was significantly higher compared to TNa (Wilcoxon $p<0.01$ ), treatment groups did not otherwise not significantly differ in their $\log _{10}$ spore loads from each other.

\section{Haemolymph sugar concentrations}

We found no significant differences in haemolymph sugar concentrations between treatment groups for glucose (Kruskal-Wallis: $\chi^{2}=3.376, \mathrm{df}=5, p=0.642$, Fig. 1a) and fructose (Kruskal-Wallis: $\chi^{2}=9.827, \mathrm{df}=5, p=0.080$, Fig. $1 \mathrm{~b}$ ). However, there was a significant interaction between honeybee lineage and treatment affecting trehalose concentrations (ANOVA: $\mathrm{F}_{2,142}=7.740, p<0.001$; Fig. $1 \mathrm{c}$ ) whereby $N$. ceranae infection was associated with reduced haemolymph trehalose availability in Nosema sensitive honeybees (SNc: 
mean $\pm 95 \% \mathrm{CI}=11.0 \pm 3.4 \mathrm{mg} / \mathrm{ml}, n=23)$ compared to tolerant honeybees (TNc: $24.9 \pm 3.4 \mathrm{mg} / \mathrm{ml}, n=27 ; p<0.001)$. Furthermore, trehalose concentrations in SNc were significantly lower compared to $\mathrm{SNa}(19.1 \pm 4.2 \mathrm{mg} / \mathrm{ml}, n=27 ; p<0.05)$ and SC $(18.4 \pm 4.3 \mathrm{mg} / \mathrm{ml}, n=25 ; p<0.05)$. Nevertheless, trehalose concentrations in TNc and TNa $(21.4 \pm 4.4 \mathrm{mg} / \mathrm{ml}, n=17)$ were not significantly different compared to their controls (TC: $19.7 \pm 2.7 \mathrm{mg} / \mathrm{ml}, n=17$; TNc-TC: $p=0.18$; TNa-TC: $p>0.05)$. There was also no significant difference of trehalose concentrations between uninfected controls of both honeybee lineages (SC, TC; $p>0.05)$.
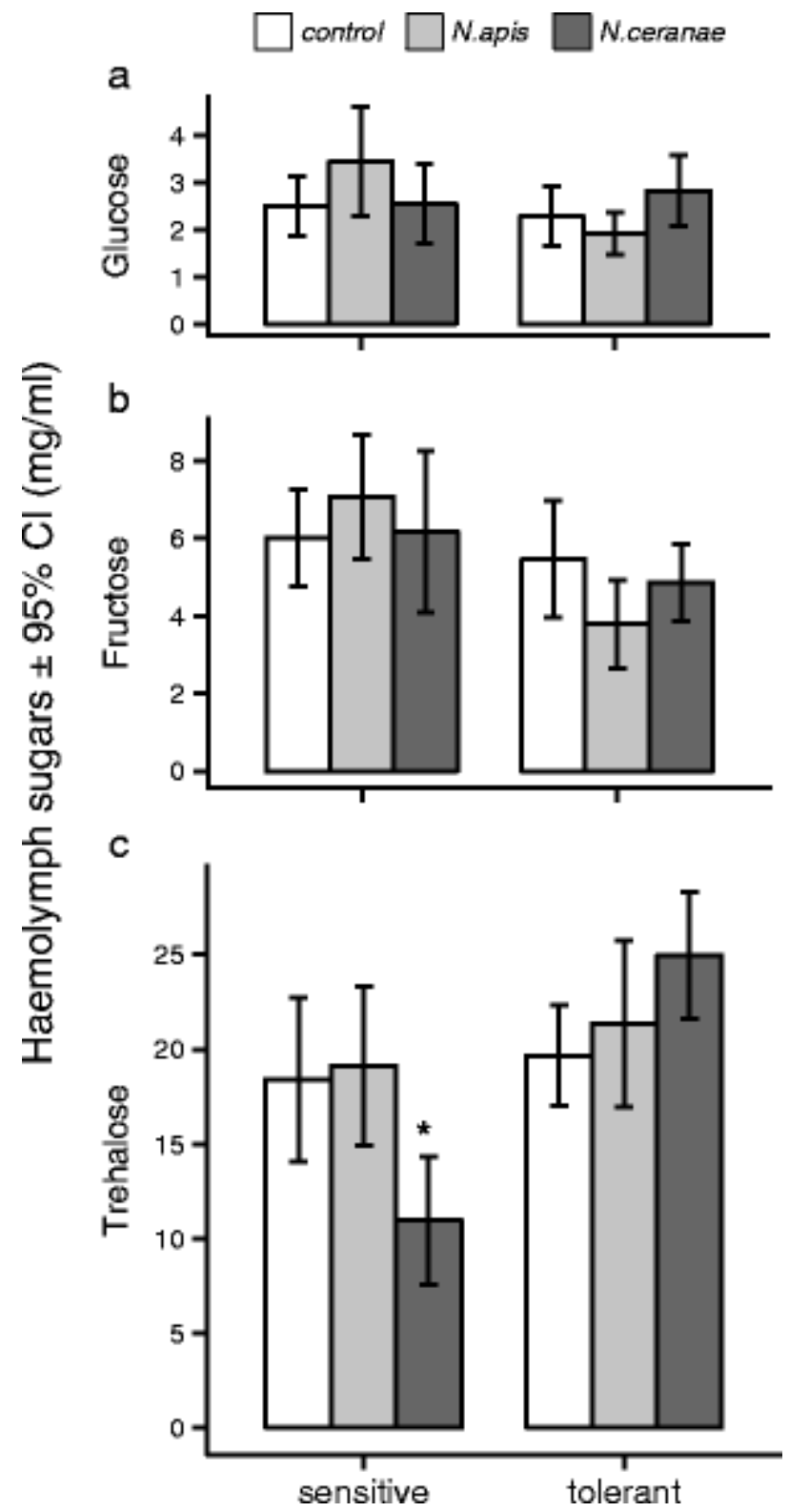

Fig 1 : Effect of Nosema spp. infection on haemolymph sugar levels in Nosema sensitive and tolerant honeybees 6 days after infection. Mean sugar concentrations $\pm 95 \%$ confidence intervals $(\mathrm{CI})$ for glucose (a), fructose (b), trehalose (c) in uninfected controls (white), N. apis (light grey) and N. ceranae (dark grey) infected sensitive (left; $n=25,27,23$ ) and tolerant strain honeybees (right; $n=29,17,27)$. Significant differences $p<0.05$ are indicated by asterisks 


\section{Regression between spore load and haemolymph sugars}

Linear regression analyses revealed a highly significant negative association between infection load and trehalose titres for $N$. apis infected sensitive honeybees (SNa: adjusted $\mathrm{R}^{2}=0.497, \mathrm{~F}_{1,18}=17.75$, s.e. of estimate $=8.729, p<0.001 ;$ Fig. $2 \mathrm{a}$ ), but not for $N$. ceranae infected sensitive honeybees ( $\mathrm{SNc}$ : adjusted $\mathrm{R}^{2}=-0.049, \mathrm{~F}_{1,20}=0.014$, s.e. of estimate $=8.159, p=0.908$; Fig. 2a). Moreover, we found neither in $N$. apis nor $N$. ceranae infected tolerant honeybees such association (TNa: adjusted $\mathrm{R}^{2}=-0.035, \mathrm{~F}_{1,13}=0.526$, s.e. of estimate $=7.793, p=0.481$; TNc: adjusted $\mathrm{R}^{2}=-0.047, \mathrm{~F}_{1,20}=0.058$, s.e. of estimate $=9.023$, $p=0.812$, Fig. 2b). The difference observed on trehalose availability was supported by a significant interaction between Nosema spp. and $\log _{10}$ spore load (MANCOVA: $F_{3}$, ${ }_{38}=10.848$, s.e. of estimate $\left.=5.043, p<0.05\right)$.

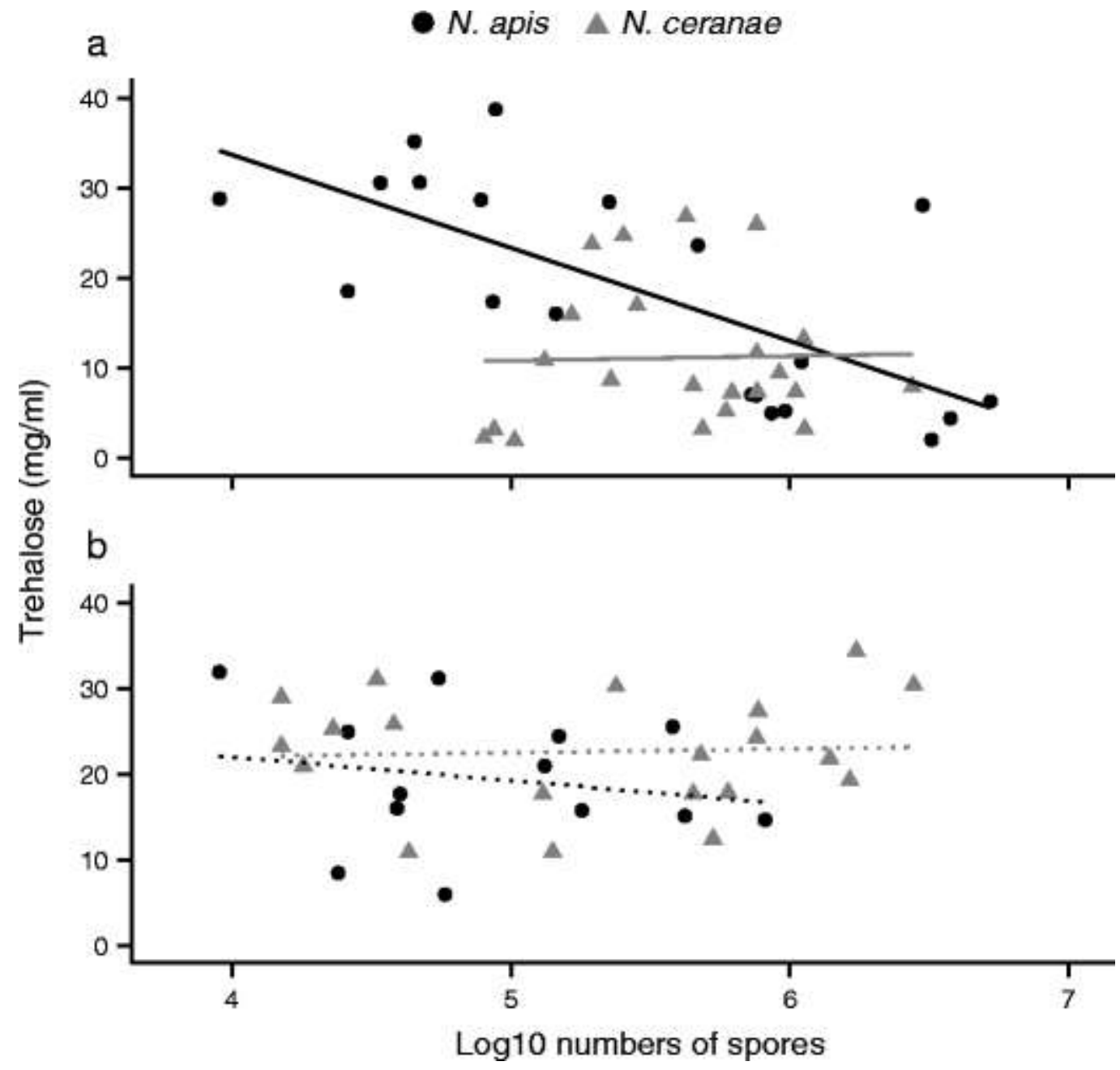

Fig 2 : Relationship between $\log _{10}$ transformed $N$. apis (black circles) and N. ceranae (grey triangles) spore loads and haemolymph trehalose concentrations in Nosema sensitive (a) and tolerant (b) honeybees after 6 days of infection. Each data point represents the measurements for a single honeybee. The lines in the graphs are regression lines: $(\mathbf{a}$, black $)$ adjusted $\mathrm{R}^{2}=0.497, \mathrm{~F}_{1,18}=17.75$, s.e. of estimate $=8.729, p<0.001 ;(\mathbf{a}$, grey $)$ adjusted $\mathrm{R}^{2}=-0.049, \mathrm{~F}_{1,20}=0.014$, s.e. of estimate $=8.159, p=0.908 ;(\mathbf{b}$, black $)$ adjusted $\mathrm{R}^{2}=-0.035, \mathrm{~F}_{1}$, ${ }_{13}=0.526$, s.e. of estimate $=7.793, p=0.481$ and $(\mathbf{b}$, grey $)$ adjusted $\mathrm{R}^{2}=-0.047, \mathrm{~F}_{1,20}=0.058$, s.e. of estimate $=9.023, p=0.812$ 


\section{Discussion}

Our study revealed that declining levels of trehalose in the haemolymph correlate with increasing $N$. apis spore load in sensitive honeybees. Although N. ceranae infections were also associated with reduced trehalose levels in sensitive honeybees, we did not find such correlation between trehalose levels and spore loads. This could possibly be explained by the limited infection range, as we have not detected any individuals from the sensitive lineage with low N. ceranae infection intensities. From our data, worker bees with medium infection loads appeared as energetically compromised as those with high infection levels. This and the fact that we did not detect workers with low spore loads in our samples may reflect a higher virulence of $N$. ceranae. On the one hand, it seems that $N$. ceranae elicits a high metabolic response at lower spore load than $N$. apis does, indicating higher energetic costs for workers to fight an infection with $N$. ceranae. On the other hand, spore load may have increased more rapidly in $N$. ceranae than in $N$. apis, which could alternatively be explained by differences between inocula. Despite this, our results would support the notion of higher virulence due to a relatively recent host-parasite relationship compared to $N$. apis, which has a long hostparasite coevolutionary history (Higes et al., 2007; Martín-Hernández et al., 2011; Williams et al., 2014). Nevertheless, our findings confirm that Nosema infections are depleting the amount of available trehalose present in the haemolymph (Mayack \& Naug, 2010), which may be responsible for the reduced survival of infected sensitive honeybees (Dussaubat et al., 2012; Higes et al., 2007).

In contrast, we found no such association over a comparable range of either $N$. apis or $N$. ceranae spore loads with trehalose levels in the haemolymph of infected honeybees of tolerant lineage. Apparently, these bees were on average able to maintain the normal energy availability as the uninfected controls. Although we cannot rule out that Nosema will not have any effect on tolerant host at much higher infection intensities, but our data may provide an explanation why their survival was not affected in inoculation experiments (Huang et al., 2012). It is important to note that a few individuals displayed relatively low trehalose levels in the haemolymph even at lower infection intensities. This observation may be explained by the intrinsic high variability of sugar levels and metabolic rates found in honeybees (Fell, 1990; Harrison \& Fewell, 2002) or alternatively this may indicate cost of immune defence in these individuals. Nevertheless, selective breeding for Nosema absence has not only resulted in genetically distinct honeybees (Huang et al., 2014a; Huang et al., 2014b) that appear to have acquired a tolerance mechanism to keep N. ceranae at bay (Kurze et al., 2015) but they also appear to maintain sufficient energy stores as well. Because suppression of the acute immune response in sensitive honeybees might be the consequence of the energetic stress imposed by a Nosema infection (Antúnez et al., 2009; Holt et al., 2013), the maintenance of high trehalose levels in Nosema tolerant honeybees might explain the preservation of immune responses found in these bees when challenged with a $N$. ceranae infection (Huang et al., 2012; Kurze et al., 2015).

Haemolymph trehalose serves as a major energy store in honeybees and other insects, providing rapid energy availability essential for flight (Suarez et al., 2005; Suarez et al., 1996; Thompson, 2003). As trehalose synthesis is energetically costly (Thompson, 2003), it is thought to be the limiting step in the maintenance of high trehalose levels in honeybees because its synthesis cannot keep up with its degradation when energy demand is high (Woodring et al., 1994). Honeybees maintain low glucose and fructose concentrations in the haemolymph at the expenses of trehalose, especially if their metabolic rates are high, like when flying during foraging trips (Blatt \& Roces, 2001). This would plausibly explain why 
we did not detect any significant alterations in glucose and fructose levels in infected honeybees relative to uninfected bees as shown by Aliferis et al., (2012). As the haemolymph concentration of glucose, fructose, and trehalose levels in uninfected control honeybees in our study were within the expected range presented in previous physiological studies (Blatt \& Roces, 2001; Woodring et al., 1993), we are confident of the reliability of our HPLC measurements and the mechanistic representation of the influence of Nosema infection on the energy demand at individual level of the bee.

As the chemosensory sensitivity of taste receptors in insects is linked with the concentration of haemolymph carbohydrates (Thompson, 2003), it is suspected that decreasing trehalose levels lead to an elevated appetite responsiveness in Nosema-infected sensitive honeybees (Mayack \& Naug, 2009). Although this would indicate increased hunger in those honeybees, Nosema-infected workers did not consume more food despite increased mortality (Williams et al., 2014). However, decreased trophallaxis (food sharing) in Nosema-infected honeybees (Naug \& Gibbs, 2009), suggests that energetically stressed honeybees are not likely to share food with their nest mates. Instead, Nosema-infected honeybees actually leave the hive energetically stressed (Mayack \& Naug, 2010) and may not return (Wolf et al., 2014). This behavioural alteration of infected honeybees might also reduce the parasite transmission and ultimately limit the damage at the colony level. However, changes in the foraging behaviour of infected individuals is also likely to negatively affect the life-history traits of the colony (Alaux et al., 2014; Naug, 2014; Wolf et al., 2014) and lower its overall fitness as a whole in the long run.

Nosema tolerant honeybees might be able to compensate the energetic losses due to the infection by modifying their behaviour in terms of food consumption or activity. Therefore, the actual energetic cost of mounting the immune response against the Nosema infection (Huang et al., 2012; Lochmiller \& Deerenberg, 2000) might not be relevant in the tolerant honeybees. This is also supported by data showing that better alimentation affected host survival positively regardless the severity of the Nosema infection (Jack et al., 2016).

Although resource allocation makes it also difficult to disentangle whether costs are caused by the parasite directly or by the immune defence (Rauw, 2012), our individual-based data suggests that the energy imbalance in the Nosema sensitive honeybee lineage is caused by Nosema spp. directly rather than by the host's immune response. Firstly, because trehalose levels were not significantly negatively affected in the infected tolerant honeybees, even though they show an increased immune response (Huang et al., 2012). This may also imply that the cost of immune response as such might be reduced as consequence of tolerance which is working alongside with an increase in efficiency of a refuelling mechanism. Secondly, microsporidia are known to be highly dependent on the ATP as energy source from the host as they can only obtain ATP themselves via glycolysis (Keeling, 2009; Williams, 2009) and we found a negative association between available trehalose in the haemolymph and the infection load of $N$. apis in sensitive honeybees.

In conclusion, our results clearly show that Nosema infection leads to an energy imbalance in sensitive honeybee workers, but not in the Nosema tolerant honeybees. These tolerant honeybees appear to have acquired a mechanism to maintain haemolymph carbohydrate homeostasis when food is available ad libitum. Possibly, this trait might play a key role in the maintenance of their immune response towards Nosema spp. and could help in explaining their advantage to fight and withstand the disease in contrast to Nosema sensitive honeybees. The energetic state might not only be important for the individual honeybee during a 
pathogen-challenge, but is also likely to affect the overall fitness of the colony as a whole. The energetic stress caused by the Nosema spp. on an individual level is therefore likely to scale-up and have major implications on the general performance of the entire colony.

\section{Acknowledgments}

We thank three anonymous reviewers for their constructive comments, which helped to improve our manuscript. The study was supported by the Deutsche Forschungsgemeinschaft DFG priority programme SPP 1399 "Host-parasite co-evolution" (grant number MO373/262).

\section{References}

Alaux C, Crauser D, Pioz M, Saulnier C, Le Conte Y (2014) Parasitic and immune modulation of flight activity in honey bees tracked with optical counters. J Exp Biol 217:3416-3424. doi:10.1242/jeb.105783

Aliferis KA, Copley T, Jabaji S (2012) Gas chromatography-mass spectrometry metabolite profiling of worker honey bee (Apis mellifera L.) hemolymph for the study of Nosema ceranae infection. J Insect Physiol 58:1349-1359. doi:10.1016/j.jinsphys.2012.07.010

Antúnez K, Martín-Hernández R, Prieto L, Meana A, Zunino P, Higes M (2009) Immune suppression in the honey bee (Apis mellifera) following infection by Nosema ceranae (Microsporidia). Environ Microbiol 11:2284-2290. doi:10.1111/j.1462-2920.2009.01953.x

Blatt J, Roces F (2001) Haemolymph sugar levels in foraging honeybees (Apis mellifera carnica): dependence on metabolic rate and in vivo measurement of maximal rates of trehalose synthesis. J Exp Biol 204:2709-2716

Bozic J, Woodring J (1997) Effect of activity on the haemolymph sugar titres in honey bees. J Apicult Res 36:33-39

Chaimanee V, Chantawannakul P, Chen Y, Evans JD, Pettis JS (2012) Differential expression of immune genes of adult honey bee (Apis mellifera) after inoculated by Nosema ceranae. J Insect Physiol 58:1090-1095. doi:10.1016/j.jinsphys.2012.04.016

Dussaubat C, Brunet J-L, Higes M, Colbourne JK, Lopez J, Choi J-H, Martín-Hernández R, Botias C, Cousin M, McDonnell C, Bonnet M, Belzunces LP, Moritz RFA, Le Conte Y, Alaux C (2012) Gut pathology and responses to the microsporidium Nosema ceranae in the honey bee Apis mellifera. Plos One 7 doi:10.1371/journal.pone.0037017

Dussaubat C, Maisonnasse A, Crauser D, Beslay D, Costagliola G, Soubeyrand S, Kretzchmar A, Le Conte Y (2013) Flight behavior and pheromone changes associated to Nosema ceranae infection of honey bee workers (Apis mellifera) in field conditions. J Invertebr Pathol 113:42-51. doi:10.1016/j.jip.2013.01.002

Fell RD (1990) The qualitative and quantitative-analysis of insect hemolymph sugars by high-performance thin-layer chromatography. Comp Biochem Phys A 95:539-544. doi:10.1016/0300-9629(90)90735-b 
Forsgren E, Fries I (2010) Comparative virulence of Nosema ceranae and Nosema apis in individual European honey bees. Vet Parasitol 170:212-217.

doi:10.1016/j.vetpar.2010.02.010

Fries I (2010) Nosema ceranae in European honey bees (Apis mellifera). J Invertebr Pathol 103:S73-S79. doi:10.1016/j.jip.2009.06.017

Fries I, Granados RR, Morse RA (1992) Intracellular germination of spores of Nosema apis Z. Apidologie 23:61-70. doi:10.1051/apido:19920107

Fries I, Chauzat MP, Chen YP, Doublet V, Genersch E, Gisder S, Higes M, McMahon DP, Martin-Hernandez R, Natsopoulou M, Paxton RJ, Tanner G, Webster TC, Williams GR (2013) Standard methods for Nosema research. J Apicult Res 52 doi:10.3896/ibra.1.52.1.14

Gisder S, Genersch E (2013) Molecular differentiation of Nosema apis and Nosema ceranae based on species-specific sequence differences in a protein coding gene. J Invertebr Pathol 113:1-6. doi:10.1016/j.jip.2013.01.004

Gisder S, Mockel N, Linde A, Genersch E (2011) A cell culture model for Nosema ceranae and Nosema apis allows new insights into the life cycle of these important honey beepathogenic microsporidia. Environ Microbiol 13:404-413. doi:10.1111/j.1462-

2920.2010.02346.x

Hamilton WD (1964) The genetical evolution of social behaviour. I J Theor Biol 7:1-16. doi:10.1016/0022-5193(64)90038-4

Harrison JF, Fewell JH (2002) Environmental and genetic influences on flight metabolic rate in the honey bee, Apis mellifera. Comp Biochem Phys A 133:323-333. doi:10.1016/s10956433(02)00163-0

Hartfelder K, Bitondi MMG, Brent CS, Guidugli-Lazzarini KR, Simoes ZLP, Stabentheiner A, Tanaka ED, Wang Y (2013) Standard methods for physiology and biochemistry research in Apis mellifera. J Apicult Res 52 doi:10.3896/ibra.1.52.1.06

Hatjina F, Bienkowska M, Charistos L, Chlebo R, Costa C, Dražić MM, Filipi J, Gregorc A, Ivanova EN, Kezić N, Kopernicky J, Kryger P, Lodesani M, Lokar V, Mladenovic M, Panasiuk B, Petrov P, Rai S, Smodis Skerl MI, Vejsns F, Wilde J (2014) A review of methods used in some European countries for assessing the quality of honey bee queens through their physical characters and the performance of their colonies. J Apicult Res 53:337-363. doi:10.3896/IBRA.1.53.3.02

Higes M, García-Palencia P, Martín-Hernández R, Meana A (2007) Experimental infection of Apis mellifera honeybees with Nosema ceranae (Microsporidia). J Invertebr Pathol 94:211217. doi:10.1016/j.jip.2006.11.001

Higes M, Meana A, Bartolomé C, Botías C, Martín-Hernández R (2013) Nosema ceranae (Microsporidia), a controversial 21st century honey bee pathogen. Environ Microbiol Rep 5:17-29. doi:10.1111/1758-2229.12024 
Holt HL, Aronstein KA, Grozinger CM (2013) Chronic parasitization by Nosema microsporidia causes global expression changes in core nutritional, metabolic and behavioral pathways in honey bee workers (Apis mellifera). BMC Genomics 14 doi:10.1186/1471-216414-799

Huang W-F, Solter LF (2013) Comparative development and tissue tropism of Nosema apis and Nosema ceranae. J Invertebr Pathol 113:35-41. doi:10.1016/j.jip.2013.01.001

Huang Q, Kryger P, Le Conte Y, Moritz RFA (2012) Survival and immune response of drones of a Nosemosis tolerant honey bee strain towards $N$. ceranae infections. J Invertebr Pathol 109:297-302. doi:10.1016/j.jip.2012.01.004

Huang Q, Kryger P, Le Conte Y, Lattorff HMG, Kraus FB, Moritz RFA (2014a) Four quantitative trait loci associated with low Nosema ceranae (Microsporidia) spore load in the honeybee Apis mellifera. Apidologie 45:248-256 doi:10.1007/s13592-013-0243-4

Huang Q, Lattorff HMG, Kryger P, Le Conte Y, Moritz RFA (2014b) A selective sweep in a microsporidian parasite Nosema-tolerant honeybee population, Apis mellifera. Anim Genet 45:267-273 doi:10.1111/age.12114

Hurd H (2001) Host fecundity reduction: a strategy for damage limitation? Trends Parasitol 17:363-368. doi:10.1016/s1471-4922(01)01927-4

Jack CJ, Uppala SS, Lucas HM, Sagili RR (2016) Effects of pollen dilution on infection of Nosema ceranae in honey bees. J Insect Physiol 87:12

Keeling P (2009) Five questions about Microsporidia. PLoS Pathog 5 doi:10.1371/journal.ppat.1000489

Kurze C, Le Conte Y, Dussaubat C, Erler S, Kryger P, Lewkowski O, Müller T, Widder M, Moritz RF (2015) Nosema tolerant honeybees (Apis mellifera) escape parasitic manipulation of apoptosis. Plos One 10:e0140174

Lochmiller RL, Deerenberg C (2000) Trade-offs in evolutionary immunology: just what is the cost of immunity? Oikos 88:87-98. doi:10.1034/j.1600-0706.2000.880110.x

Martín-Hernández R, Botías C, Barrios L, Martinez-Salvador A, Meana A, Mayack C, Higes M (2011) Comparison of the energetic stress associated with experimental Nosema ceranae and Nosema apis infection of honeybees (Apis mellifera). Parasitol Res 109:605-612. doi:10.1007/s00436-011-2292-9

Mayack C, Naug D (2009) Energetic stress in the honeybee Apis mellifera from Nosema ceranae infection. J Invertebr Pathol 100:185-188. doi:10.1016/j.jip.2008.12.001

Mayack C, Naug D (2010) Parasitic infection leads to decline in hemolymph sugar levels in honeybee foragers. J Insect Physiol 56:1572-1575. doi:10.1016/j.jinsphys.2010.05.016

Moffett JO, Lawson FA (1975) Effect of Nosema-infection on O2 consumption by honey bees. J Econ Entomol 68:627-629 
Moret Y, Schmid-Hempel P (2000) Survival for immunity: the price of immune system activation for bumblebee workers. Science 290:1166-1168.

doi:10.1126/science.290.5494.1166

Moritz R, Southwick EE (1992) Bees as superorganisms: an evolutionary reality. Springer, Berlin Heidelberg

Naug D (2014) Infected honeybee foragers incur a higher loss in efficiency than in the rate of energetic gain. Biol Lett 10 doi:10.1098/rsbl.2014.0731

Naug D, Gibbs A (2009) Behavioral changes mediated by hunger in honeybees infected with Nosema ceranae. Apidologie 40:595-599. doi:10.1051/apido/2009039

R Development Core Team (2015) R: a language and environment for statistical computing. R Foundation for Statistical Computing, Vienna, Austria, http://www.R-project.org/ . ISBN 3-900051-07-0

Råberg L, Sim D, Read AF (2007) Disentangling genetic variation for resistance and tolerance to infectious diseases in animals. Science 318:812-814.

doi:10.1126/science. 1148526

Rauw WM (2012) Immune response from a resource allocation perspective. Front Genet 3:267. doi:10.3389/fgene.2012.00267

Roth O, Kurtz J (2008) The stimulation of immune defence accelerates development in the red flour beetle (Tribolium castaneum). J Evol Biol 21:1703-1710. doi:10.1111/j.14209101.2008.01584.x

Sadd BM, Siva-Jothy MT (2006) Self-harm caused by an insect's innate immunity. P Roy Soc B-Biol Sci 273:2571-2574. doi:10.1098/rspb.2006.3574

Schmid-Hempel P (2005) Evolutionary ecology of insect immune defenses. Annu Rev Entomol 50:529-551. doi:10.1146/annurev.ento.50.071803.130420

Schmid-Hempel P (2008) Parasite immune evasion: a momentous molecular war. Trends Ecol Evol 23:318-326. doi:10.1016/j.tree.2008.02.011

Sheldon BC, Verhulst S (1996) Ecological immunology: costly parasite defences and tradeoffs in evolutionary ecology. Trends Ecol Evol 11:317-321. doi:10.1016/01695347(96)10039-2

Sorci G (2013) Immunity, resistance and tolerance in bird-parasite interactions. Parasite Immunol 35:350-361. doi:10.1111/pim.12047

Straub L, Williams GR, Pettis J, Fries I, Neumann P (2015) Superorganism resilience: eusociality and susceptibility of ecosystem service providing insects to stressors. Curr Opin Insect Sci 12:109 
Suarez RK, Lighton JRB, Joos B, Roberts SP, Harrison JF (1996) Energy metabolism, enzymatic flux capacities, and metabolic flux rates in flying honeybees. Proc Natl Acad Sci U S A 93:12616-12620. doi:10.1073/pnas.93.22.12616

Suarez RK, Darveau CA, Welch KC, O’Brien DM, Roubik DW, Hochachka PW (2005) Energy metabolism in orchid bee flight muscles: carbohydrate fuels all. J Exp Biol 208:35733579. doi:10.1242/jeb.01775

Thompson SN (2003) Trehalose - the insect 'blood' sugar. In: Simpson SJ (ed) Adv Insect Physiol, Vol 31, vol 31. Advances in Insect Physiology. pp 205-285. doi:10.1016/s00652806(03)31004-5

Williams BAP (2009) Unique physiology of host-parasite interactions in Microsporidia infections. Cell Microbiol 11:1551-1560. doi:10.1111/j.1462-5822.2009.01362.x

Williams GR, Shutler D, Burgher-MacLellan KL, Rogers REL (2014) Infra-population and community dynamics of the parasites Nosema apis and Nosema ceranae, and consequences for honey bee (Apis mellifera) hosts. Plos One 9 doi:10.1371/journal.pone.0099465

Wolf S, McMahon DP, Lim KS, Pull CD, Clark SJ, Paxton RJ, Osborne JL (2014) So near and yet so far: harmonic radar reveals reduced homing ability of Nosema infected honeybees. Plos One 9 doi:10.1371/journal.pone.0103989

Woodring J, Boulden M, Das S, Gade G (1993) Studies on blood-sugar homeostasis in the honeybee (Apis mellifera, L). J Insect Physiol 39:89-97. doi:10.1016/0022-1910(93)90022-j

Woodring J, Das S, Gade G (1994) Hypertrehalosemic factors from the corpora cardiaca of the honeybee (Apis mellifera) and the paper wasp (Polistes exclamans). J Insect Physiol 40:685-692. doi:10.1016/0022-1910(94)90095-7 